average (MMA) method to assess the utility of $\mathrm{T}$-wave alternans (TWA) and heart rate turbulence (HRT) as risk markers in MI patients with or without diabetes mellitus (DM).

Methods The study population included 248 patients: 96 with MI (group post-MI); $77 \mathrm{MI}$ with DM (group post-MI+DM); 75 controls (group control). Both TWA and HRT were measured on ambulatory ECG (AECGs). TWA $(>47 \mu \mathrm{V})$ was considered abnormal. HRT was assessed by two parameters-turbulence onset (TO) and turbulence slope (TS), and HRT values were classified into three categories. HRT was considered positive when both $\mathrm{TO} \geq 0 \%$ and TS $\leq 2.5 \mathrm{~ms} / \mathrm{R}-\mathrm{R}$ interval were met. The endpoint was cardiac mortality.

Results T-wave alternans differed significantly between controls and post-MI groups with $(37 \pm 13 \mu \mathrm{V}$ VS $58 \pm 21 \mu \mathrm{V}, \mathrm{p}<0.05)$ or without DM $(37 \pm 13 \mu \mathrm{V}$ VS $52 \pm 18 \mu \mathrm{V}, \mathrm{p}<0.05)$, and compared to post-MI group, group post-MI+DM had a higher TWA values $(p=0.029)$. Average values for TO and TS differed significantly between controls and the two post-MI groups $(p<0.05)$. Groups post-MI with or without DM had a higher association of positive results for both TWA $(>47 \mu \mathrm{V}$ ) and HRT (TO $\geq 0 \%$ and TS $\leq 2.5 \mathrm{~ms} / \mathrm{RR}$ ). During follow-up of $578 \pm 146$ days, cardiac death occurred in 10 patients, and two of them suffered witnessed sudden death. Multivariate analysis determined that a HRT-positive outcome (HR (95\% CI) 5.01, 1.33 to 18.85; $p=0.017$ ) and the combination of abnormal TWA and positive HRT had significant association with the endpoint (HR (95\% CI) 9.08, 2.21 to 37.2; $\mathrm{p}=0.002)$ ).

Conclusions TWA values in post MI with DM was higher than post MI. Impaired HRT, increased TO and decreased TS were observed in MI patients with or without DM. TWA and HRT may be useful predictors of cardiac and arrhythmic death in MI patients.

\title{
Non-invasive cardiac electrical inspection GW23-e0085 AMBULATORY ECG-BASED T-WAVE ALTERNANS AND HEART RATE TURBULENCE IN PATIENTS WITH MYOCARDIAL INFARCTION WITH OR WITHOUT DIABETES MELLITUS
}

doi:10.1136/heartjnl-2012-302920t.1

Ren Li-Na, Fang Xin-Hui, Wang Yong-quan, Qi Guo-xian. Department of Cardiology, the First Affiliated Hospital of China Medical University, Shenyang

Objectives Many patients who survive a myocardial infarction (MI) remain at risk of sudden cardiac death despite revascularisation and optimal medical treatment. We used the modified moving 